\title{
User Experience of Markerless Augmented Reality Applications in Cultural Heritage Museums: 'MuseumEye' as a Case Study
}

\author{
Ramy Hammady ${ }^{\mathrm{a}}$, Minhua $\mathrm{Ma}^{\mathrm{a}}$, and Anna Powell ${ }^{\mathrm{b}}$ \\ ${ }^{a}$ School of Computing and Digital Technology \\ Staffordshire University, United Kingdom \\ ramy.hammady@research.staffs.ac.uk \\ m.maestaffs.ac.uk \\ ${ }^{\mathrm{b}}$ School of Art, Design and Architecture \\ University of Huddersfield, United Kingdom \\ A.Powell@hud.ac.uk
}

\begin{abstract}
This paper explores the User Experience (UX) of Augmented Reality applications in museums. UX as a concept is vital to effective visual communication and interpretation in museums, and to enhance usability during a museum tour. In the project 'MuseumEye', the augmentations generated were localized based on a hybrid system that combines of (SLAM) markerless tracking technology and the indoor Beacons or Bluetooth Low Energy (BLE). These augmentations include a combination of multimedia content and different levels of visual information that required for museum visitors. Using mobile devices to pilot this application, we developed a UX design model that has the ability to evaluate the user experience and usability of the application. This paper focuses on the multidisciplinary outcomes of the project from both a technical and museological perspective based on public responses. A field evaluation of the AR system was conducted after the UX model considered. Twenty-six participants were recruited in Leeds museum and another twenty participants in the Egyptian museum in Cairo. Results showed positive responses on experiencing the system after adopting the UX design model. This study contributes on synthesizing a UX design model for AR applications to reach the optimum levels of user interaction required that reflects ultimately on the entire museum experience.
\end{abstract}

Keywords: User Experience, Markerless, UX Design Model, iBeacon, Cultural Heritage

Index Terms: [UX]: User Experience—-[MAR]: Markerless Augmented Reality

adfa, p. 1, 2011

(C) Springer-Verlag Berlin Heidelberg 2011 


\section{Introduction}

Augmented Reality (AR) as a term has evolved through the last decade [1] [2] [3]. It has become a promising trend and its potential role in museums has become more widely understood as a part of developments in approaches to museum interpretation [4] [5] [6]. With a view to addressing usability for interpretation devices in the museum context, this research has developed a mobile museum guide named 'MuseumEye' that can be employed in museums with a markerless tracking technique. The technique enables the viewer to observe objects and artefacts safely, and in more detail, outside the confines of their glass vitrines. This technique does not require a label or tag to trigger the augmentations [7]. Also, the tracking method used is an integration between MAR and indoor location beacons. The design philosophy here is primarily one of visual communication and user experience. Primary research was conducted in museums to enable the researchers to experience and test their products in an appropriate environment. The factors measured included usability, flexibility and human interactions with the technology. The literature addressed in this research supported identification of the most appropriate technical aspects of the project relevant to tracking systems, for example the functionality of Markerless tracking and the SLAM method. Ultimately, it is hoped that this investigation of user experience will provide a useful model or taxonomy transferrable to other AR applications.

Simultaneous Localization And Mapping (SLAM) is a method that can detect a model's edges from a series of images, without the need for previously identifying the real environment [8]. It is also defined as the process of simultaneously building a static map and estimating the sensor motion [9]. SLAM as a technique is illustrated when a computer with a live camera is attached to it, transforming it into a real-time sensor position [10]. It is also defined as a self-mapping of visual features which are located in the surrounding environment, through a continuous calculation of the camera motions [10]. Other researchers further evolved SLAM by defining a new algorithm that can overcome the challenge of selecting the landmarks' edges [11]. Most Augmented Reality (AR) applications that deploy the mobile devices rely on the recognition of unseen physical objects in the real environment to apply the augmentation in the process. Moreover, the tracking should be accurate and allow the user to interact with the augmentation. Most of the conventional AR applications were built on the basis of the existing marker. However, there are various limitations to the fiducial or visual markers: For instance, the superimposed objects will be lost in tracking if the camera moves fast due to the correlation of the framerate. Visual markers have a limited range for tracking and should remain in sight [8]. Furthermore, they are not scalable for the outdoor environment [12].

SLAM relies on the Markerless Augmented Reality (MAR) as it breaks the boundaries of allocating tags or images on the augmentation area. Markerless tracking has a great impact upon AR user experience. It enhances user interaction and perception with the real world without physical constrains [15]. It can consider any part of the real environment as a marker to position the virtual objects [15]. This tracking system could be achieved using various sensors technologies such as gyroscopes, hybrid vision, GPS triggering, infrared tags or triggers [16] [17] [2]. There has been a considerable amount 
of tracking research conducted with the monocular camera [18] [10] or with Kinect cameras [19] in order to track complex 3D models in real-time while avoiding occlusion and illumination [15].

There are examples of MAR tracking applications in the museums and galleries sector: A group of scholars created a project named "MapLens" [20] and it is claimed that the method used was Markerless. However, it works by identifying a paper map which has to be facing the user's camera. A further project named "ANR GAMME" was built, based on an iterative design method to guide visitors in museums, which relies on a Markerless tracking. Visitors were, however, required to angle the device's camera towards the painting on the wall to reveal the pre-prepared content [21]. Headsets such as optical see-through displays have also been used in MAR, in the context of cultural heritage, to identify the motivations and needs of museum professionals [22].

In 'MuseumEye', we resolved that one of the most efficient ways to build successful communication between the user's device and our servers was to use IBeacons. These beacons rely on using a low energy connection. The rationale behind using beacons is the large range of connection it usually has compared with infrared tags. It connects with devices via Bluetooth transmission in a range of 70 meters [23]. Bluetooth connections can influence devices within their sphere of connections, rather than relying on other ways of connection that require point-to-point correspondence [16]. So MuseumEye contributes on creating a hyper system that combines between creating MAR augmentations on SLAM technology and the indoor location beacons. This system aims to achieve robust connections between the user and the generated visuals.

This study also investigates the user experience concepts and criteria that should be taken into account during the development of AR application in museums. The investigations included a study on the tour requirements and the way the visitor responds during the usage of the AR system. These investigations contributed on creating the UX design model, that able to assist developers to create museum AR applications with enhanced usability aspects, which reflects ultimately on the museum experience.

\section{$1.1 \quad$ User Experience (UX) and (MAR)}

User Experience (UX) as a term emerged from two decades in the community of Human Computer Interaction (HCI) [24]. UX generally includes several disciplines such as visual design, human factors, information delivery, hologram design, content, sound design, and interaction design, as depicted in Figure 1. User experience was defined by ISO as a "person's perceptions and responses resulting from the use and/or anticipated use of a product, system or service". It also observes the responses ranging from emotions, perceptions, behaviours to the physical and psychological responses from users prior, during and after experiencing the application [25]. However, researchers and writers have attempted to define UX from different perspectives. For instance, Ellis defines UX as the discipline that instructs the way of changing the product in order to affect the user's feelings and his/her behaviour. It includes the whole experience of using the product/system such as making assessments about user 
preferences, even identifying moments of delight or frustration [26]. However, Unger and Chandler [27] defined user experience design within a broader definition, which is "The creation and synchronization of the elements that effect the user's experience with a particular company, with the intent of influencing their perceptions and behaviour" [27]. These are elements that are manifested in the human senses, such as touch (haptics), hearing and smell. It was difficult for the researchers and designers working in the field to decide upon a definitive definition until Law et al. surveyed 5 definitions. These definitions vary depending on the perspective of the respondent and their nature of work; either academic or industrial, and the scope of their work [24]. Based on the capacity and nature of this system, and the researcher disciplines and backgrounds, this paper uses the following definition: " $U X$ is a consequence of a user's internal state (predispositions, expectations, needs, motivation, mood, etc.), the characteristics of the designed system (e.g. complexity, purpose, usability, functionality, etc.) and the context (or the environment) within which the interaction occurs (e.g. organizational/social setting, meaningfulness of the activity, voluntariness of use, etc.)" [28]. In short, UX surpasses the traditional line of usability and achieving the product goals through creating emotional and perception-based bonds between the human and the product, whether a tangible or nontangible product.

The recent trend of mobile AR applications takes many forms such as head mounted displays, smartphones and tablets. One of the significant objectives of this paper is to discover which elements of user experience should be embraced in the proposed markerless augmented reality application, and to build a UX design model which can be used as a workflow to build successful MAR applications that foreground user experience.

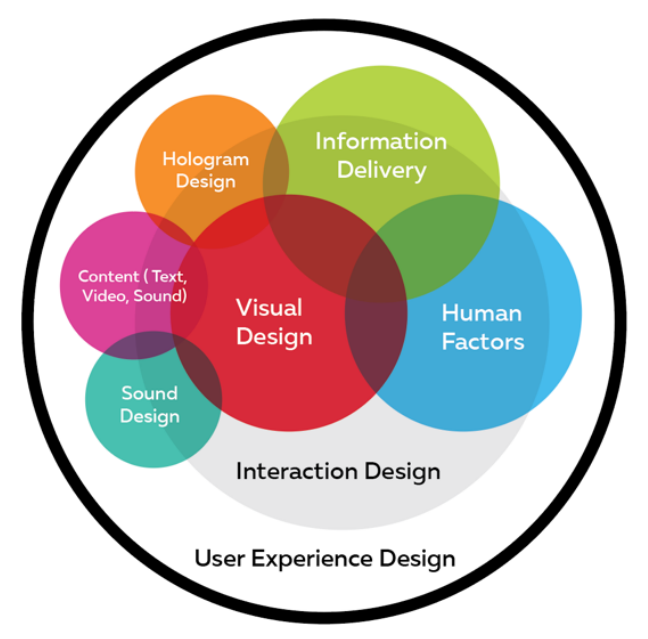

Figure 1: Diagram of User Experience involving cross disciplines (modified from www.interaction-design.org) 


\section{UX Design Process Model}

While it is essential to build a workflow that includes all design phases, it proved to be a challenge to articulate the true impact of user experience of the markerless AR application. Due to its novelty in the industry, it was difficult to identify what users want in these applications, and whether they are effective in enhancing the viewing experience. Identifying the user experience in the interactive system is even more complicated than in the traditional systems [29]. The next step, therefore, was to brainstorm a unified workflow that could work as a model for the UX design process and include these (Figure. 2). This model has three phases starting with the design requirements for UX and ended with evaluation.

In the 'requirement phase', there are some information, skills and data needed to be obtained before starting to design and develop the system. The objectives of the system had to be considered alongside the practicalities of building the system, such as providing guidance and visual information to support and not conflict with the museum's existing displays and interpretation resources. Besides this, it was necessary to acquire the information content for the artefacts with which the technology would be used, such as the 3D models, the audio commentaries, relevant images and the existing explanatory text provided alongside the exhibits. The research then took a considerable step forward to address following questions:

- Is this application intuitive and easy to use?

- Does the navigation meet expectations? In another words, is the next move anticipated?

- Are the visuals supported and can they achieve the goals set?

- Is the 3D navigation intuitive?

- Is placing the $3 \mathrm{D}$ artefacts within the physical environment intuitive or cumbersome to the viewer's experience and understanding of the exhibits?

- Does the application construct an emotional bond between it and the user so we guarantee the continuity of using it?

- Is the user satisfied and interested to continue using the app alongside his tour? 


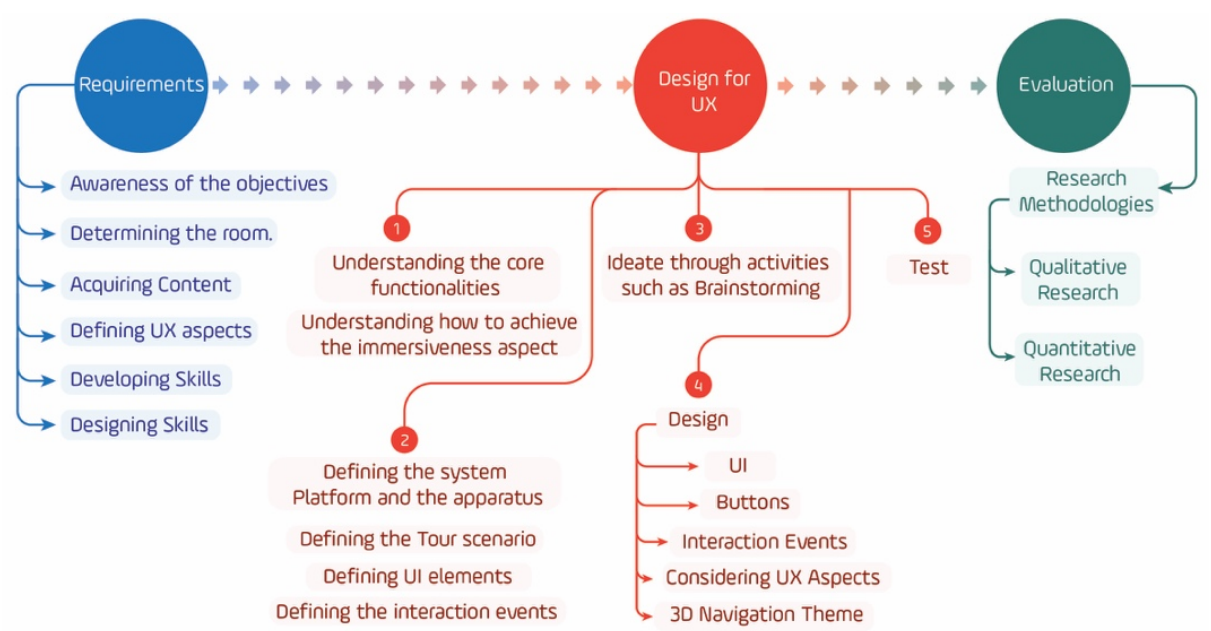

Figure 2: UX Design Process Model

Würstl believed that user experience generally comprises three main areas, 'Usability', 'Look' and 'Feel', and he suggested that usability is one third of these divisions [30]. In the following model we conclude that the UX is a mixture of usability aspects blended with the sensory and emotional aspects. By responding to the previous questions and through first hand research, we were able to devise the user experience aspects of markerless augmented reality applications depicted in Figure 3.0. In short, it has two main categories: the 'Utilitarian' aspects which focus on the main functionalities of the system, and the 'Visual' aspects that concern the appearance of the application and ways of influencing the look and feel of the system to the user. Regarding the 'Utilitarian' aspect, it has two main sections: functionality and interaction. The 'Functionality' section manifests the focus of the system and the main role that the system plays. The MAR app requires a seamless integration of the $3 \mathrm{D}$ object in the physical environments to enable the user to have the illusion of being immersed in a mixed environment. It is essential for it to be a lightweight application that does not use too much RAM, and it is recommended that it should consume enough energy from the mobile battery to last the duration of the visit. Moreover, the registration of the $3 \mathrm{D}$ objects in the real world should be very accurate to help create a sense of their realness. Customization is a key factor in letting the user feel ownership of the application which, in turn, helps them to build an emotional bond with the system. 


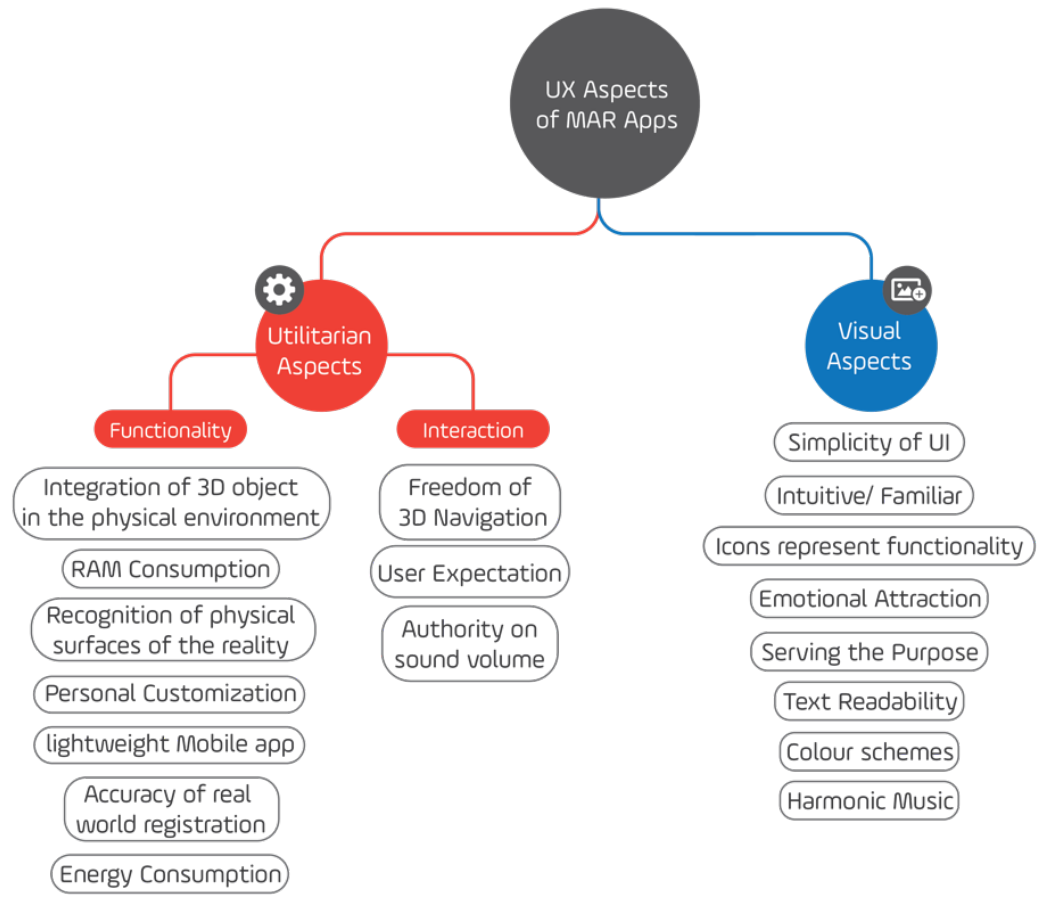

Figure 3: UX aspects of markerless augmented reality applications

The second section includes the 'Interaction' elements. These are concerned with how the user interact with the system and the responds to the system. It allocates the human to the center of the system and it also considers them to be the most integral part of the communication process between him/her and the system. These aspects necessitate giving the user the privilege of freedom of navigating the $3 \mathrm{D}$ object in the physical environment, as the user holds the object in reality. This allows the user to reposition, rotate and rescale the object. This feature was a fundamental reason behind building the application, due to the fragility of many artefacts and the simultaneous benefits to the viewer of being able to access them close at hand. One of the key usability factors of the system is to allow the user to anticipate the next click/move. It is also important to allow the user some authority over his/her device while the application is running, such as controlling the sound volume, pausing, playing and stopping the file.

Concerning the second section, the 'Visual aspects', the main objective is to construct the emotional bond between the system and the users. To achieve these objectives, it is important to make the user interface as simple as possible, including all UI elements such as buttons, headers, images etc. Familiarity is one of the key aspects in creating an emotional bond between the user and the application; to create the sense that they have used the application before. The idea behind the abstract graphic icon was to represent its function directly without confusing the viewer. This will help to save time when using the device, and make the application more familiar and intuitive to the new user. Design skills are an important part of ensuring the UI achieves an 
emotional bond in the viewer and that it serves its intended purpose. Some elements require specific consideration to fulfil their role successfully, such as the font size and colours, which can determine the effectiveness of information delivery. Moreover, the colour scheme should be chosen in a way that is relevant to the context of the application, and the same consideration should be applied to the chosen background music for the application, which plays constantly while presenting content.

The second phase, 'Design for UX', came directly after determining the perquisites of the app. This phase built on the process of design thinking and was developed to fit the integration of user experience with the entire process.

Design for UX starts with gaining a comprehensive understanding of the context in which it will be used (the museum), and the methodology was to build system specifically targeted for this context. This step was accomplished by exploring the museum and talking to museum visitors as an exploratory study. Further knowledge was gained through referring to relevant literature about designing effective museum interpretation, and further research into the technical information of markerless augmented reality. It was also important to gain a full understanding of the objectives of the system and to understand the importance of UX as previously discussed.

The final phase is to define the specifics of certain elements of the system, such as the platform - either android or IOS - in order to prepare the developing tools that fit the targeted device, which can mount the potential system. Identifying the UI elements is important in the designing phase in order to set the interaction events and determine how the system responds to the user. Eventually, this phase ended with drawing the tour scenario for the targeted room, which will be discussed in the next section.

After gaining sufficient knowledge, the next step is to generate ideas. There are some strategies to follow to produce ideas such as brainstorming, SCAMPER [31], Brainwrite, Braindump etc [32]. These techniques are not only for generating ideas; they can reveal solutions to potential problems. Usually, this stage analyses observations and the information collected in the final stage.

The designing phase is the stage that involves sketching and designing the UI of the application including buttons, and interaction events. During this phase, all of the UX aspects will be addressed and considered. This stage should culminate in a prototype with full functionality and without bugs or errors. Moreover, this prototype should communicate perfectly with the IBeacons in order to mount them in the museum room.

The final stage is testing and this stage is conducted several times in the lab and by both designers and developers in order to reveal any flaws and errors. However, this stage is usually conducted iteratively. Whenever a problem pops up during testing, the system will be taken back to previous stages to get a full understanding of the situation, in order to determine a solution.

The third phase is 'Evaluation' and this phase involves visitors in museums and experts in the field providing valuable feedback. This stage follows a particular methodology to obtain the required feedback and it is divided into two methods: Qualitative and Quantitative research. The workflow of the evaluation phase will be demonstrated in the participation and experiments section. 


\section{3 'MuseumEye' Project}

This project used the SLAM markerless-tracking technique to achieve the most effective way of viewing appropriate to the required augmentation. Most markerless applications currently in the market depend on pointing the device's rear camera to an exhibited image. These images are considered markers to the camera and trigger the augmentations in the AR system to be revealed. Research into the development of augmented reality is now considering similar techniques to marker-tracking, but without using labels or illustrated markers. SLAM markerless tracking relies on placing the augmentations on a surface, requiring the user to point the device's camera to a further surface in order reveal a virtual object or character. The MuseumEye system considers the surface as the origin point to the object, so augmentations look as if they are placed in the real environment and have a realistic appearance.

Some of the tools that used to help us to build MuseumEye included 'Unity', and open source codes such as 'open cvs' and SDK files such as 'ARCore'. These codes and SDKs provide our system with image processing functions such as recognizing and tracking surfaces. Afterwards, the system integrates with the iBeacons which are working as servers for the mobile devices. These beacons are able to allocate the visitor's location then trigger his/her device to be fed with the augmentations, visual and haptic information needed that relevant to the antique's location.

Regarding the 3D antiques, we conducted 3D scanning for the replicas of the antiques then we obtained visual and audio data about the collected antiques. UI designed using Adobe Illustrator and other image editing software such as Adobe Photoshop.

One of the objectives of creating this system was to give the visitor the opportunity to explore a 360-degree view of the exhibited artefact, outside the confines of the glass vitrine, instead of being limited to a predetermined visual perspective. The visitor is given the freedom to see the exhibit from different perspectives, and the navigation tool added to the system allows the user to reposition, rotate and scale the object.

\subsection{Scenario}

The tour design for Leeds museum and the Egyptian museum was created to be a thematic type of tour. It was designed by synchronizing the audio content with the relevant artefacts, to develop an ambient music, which was appropriate to the museum context. Firstly, as seen in Figure 4, we drew a sensible route in the targeted rooms in both museums, one which would be a closed route to start and end at the same point in a circle shape. Then, as seen in Figure 5, we designed the tour by adding the visual and audio commentaries fitting the context of the room to make the visitor embrace the virtual content in an interesting manner. As the visitor arrives at the first point, the aim is that he/she will gain the full experience of the AR system. 


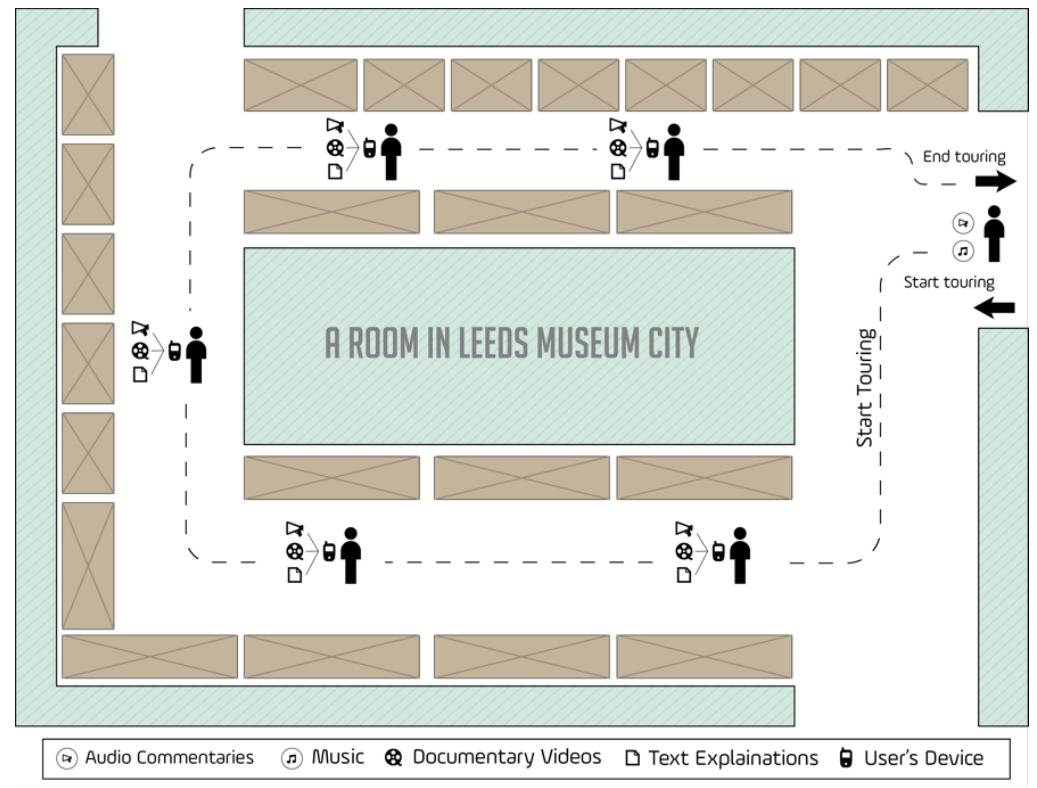

Figure 4: A planned walk Integrated with multimedia content during the visit in Leeds Museum City's room.

\section{$4 \quad$ Method}

Briefly, the method embraced for the evaluation process is constituted into two stages, the first stage is exposing the system 'MuseumEye to be tested through Leeds museum. During the experiment, an in direct observation was conducted to see the user's ability to use the system and also the observations extended to target their emotions about the usability factor. 26 from Leeds Museum's visitors were surveyed and the observation outcomes where taken into consideration to amend the UI functions in the next stage. The second stage was conducted at the Egyptian museum in Cairo and 20 participants were employed to test the system for measuring the usability and the user experience.

This following section describes the device that was adopted to operate the AR guided system alongside the experiment settings in both museums. Further, we demonstrate the experiments carried out within the museum conditions, and how the data collection was conducted in details. 


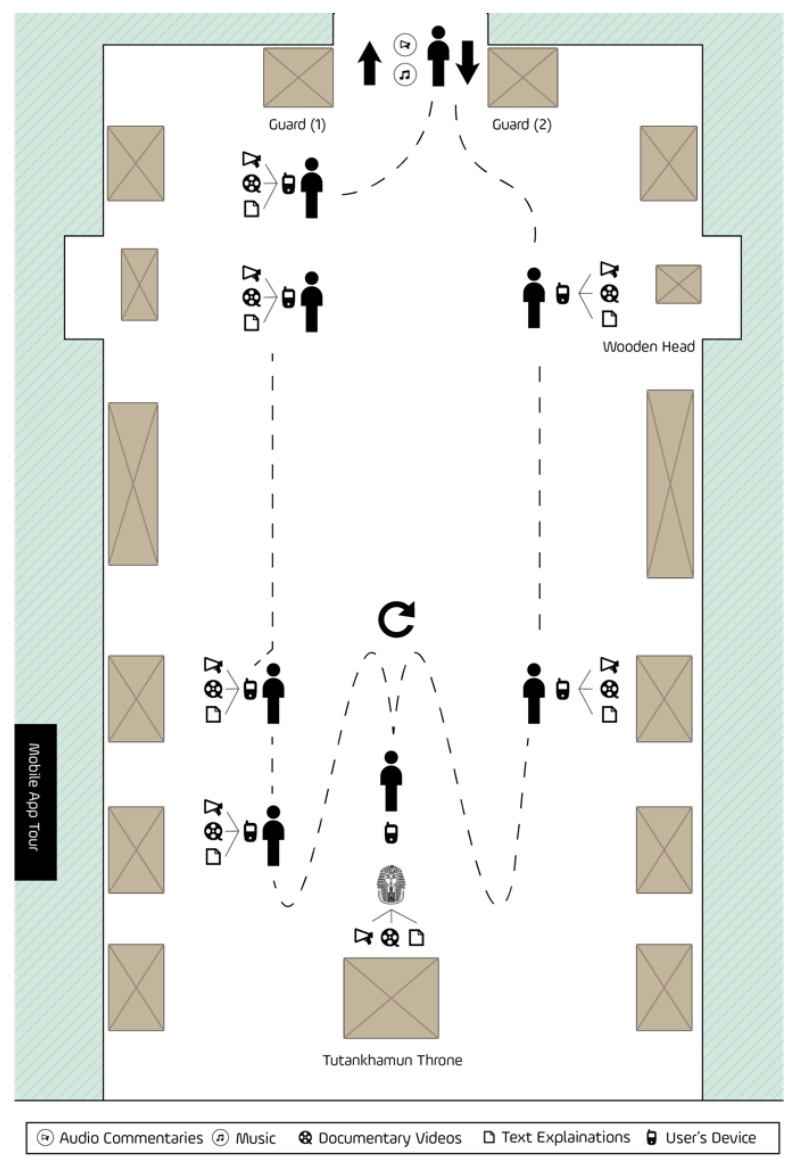

Figure 5: A planned walk Integrated with multimedia content during the visit in the Egyptian museum's room.

\subsection{Apparatus}

Our system benefits from the scalability of devices that can be used to run it. Thus, any android device higher than Android Marshmallow 3.3 can operate our system. The device employed to run our system was Amazon Fire tablet - a cheap device with many advantages. It has $1.3 \mathrm{GHz}$ quad-core processor and rear- and front-facing cameras and unlimited storage with cloud space or with the SD card. It has a display with resolution of (171 PPI / 1024 x 600).

\subsection{Software}

'MuseumEye' was built to work as a markerless application which has the benefit of being able to localize the augmented content, such as 3D models of the artefacts, in a pre-determined location. It was not possible to place markers in the museum halls due 
to the strict standards of the museum settings. It would, in any case, have been cumbersome for users to follow the markers for each item. Thus, markerless-based tracking gives the user the ability to locate the augmentation wherever he/she desires. Moreover, it has the ability to present the augmentations in real-time in the museum's environment.

Once the visitor arrives to the antique's location, the visitor gets notification from the iBeacon servers to open the interface of the application, which brings a live feed camera in front. This interface includes buttons on left and right that comprised of all desired visuals that visitor might needs such as textual, images, audio recordings. At the bottom- middle of the UI, a button requesting the visitor to allocate the virtual antique on the ground. So, the visitor can aim to the ground then presses this button, then the antique's augmentation will be placed on the ground with synced audio narratives about the antique. The most interesting part of the augmentations is the freedom of navigation of the chosen object. The user can interact with the $3 \mathrm{D}$ object by rotating, repositioning, scaling it with the proper size he/she desires, as depicted in Figure 6.

'MuseumEye' encompasses of a combination of multimedia content such as audio commentaries, video representations, and different levels of information about the selected item in a text format and a gallery of images. The user is centred in a multimedia panel which acts as an encyclopaedia to assist the visitor in gaining further information. The localization feature was executed in our system by employing IBeacons in the walls of the hall which was chosen for conducting the field-research. These beacons located the users' devices by triangulation, by sending and receiving

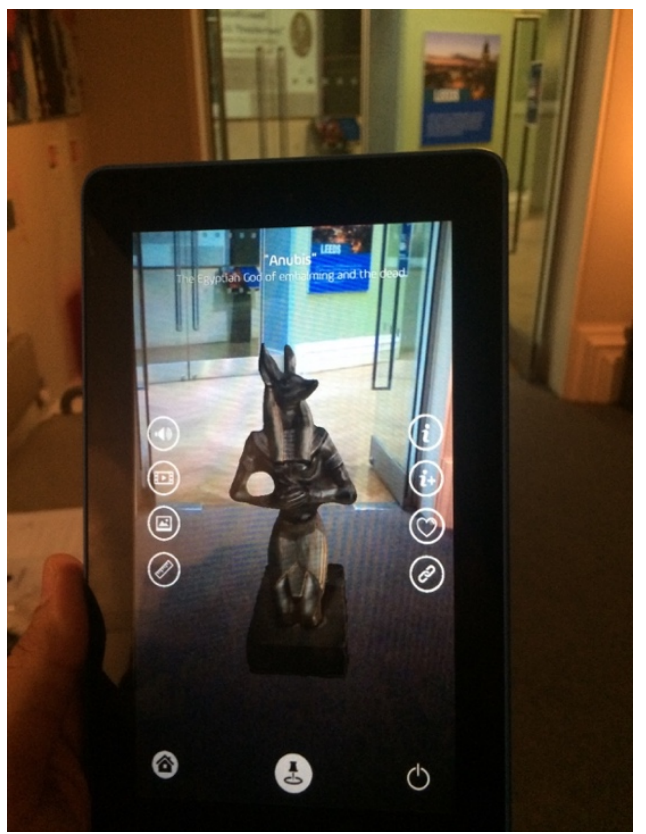

Figure 6: 'MuseumEye' places objects using MAR technology 
signals to a specific point. From this point, the user's device starts to receive the bundle of information (2D /3D visualization/video /animation). Depicted in Figure 7 is the method of employing IBeacons in Leeds City Museum and The Egyptian Museum in Cairo.

\subsection{Participants and experiments}

To identify appropriate methods for exploring and testing our system, it was first divided into qualitative and quantitative data, based on the information needed. The quantitative research relied on using quite a large sample of users to identify trends and draw conclusions. Moreover, the qualitative research is needed to get a more in-depth understanding of the context and to acquire valuable information for improving the system. At 'Stage 1' at Leeds city Museum, twenty-six participants were employed for our experiment. What distinguished that sample was the variation of their ages, they were starting from 16 years old to above 60 . There was also variation in their levels of education. This varied sample enriched our research findings. The plan was to instruct

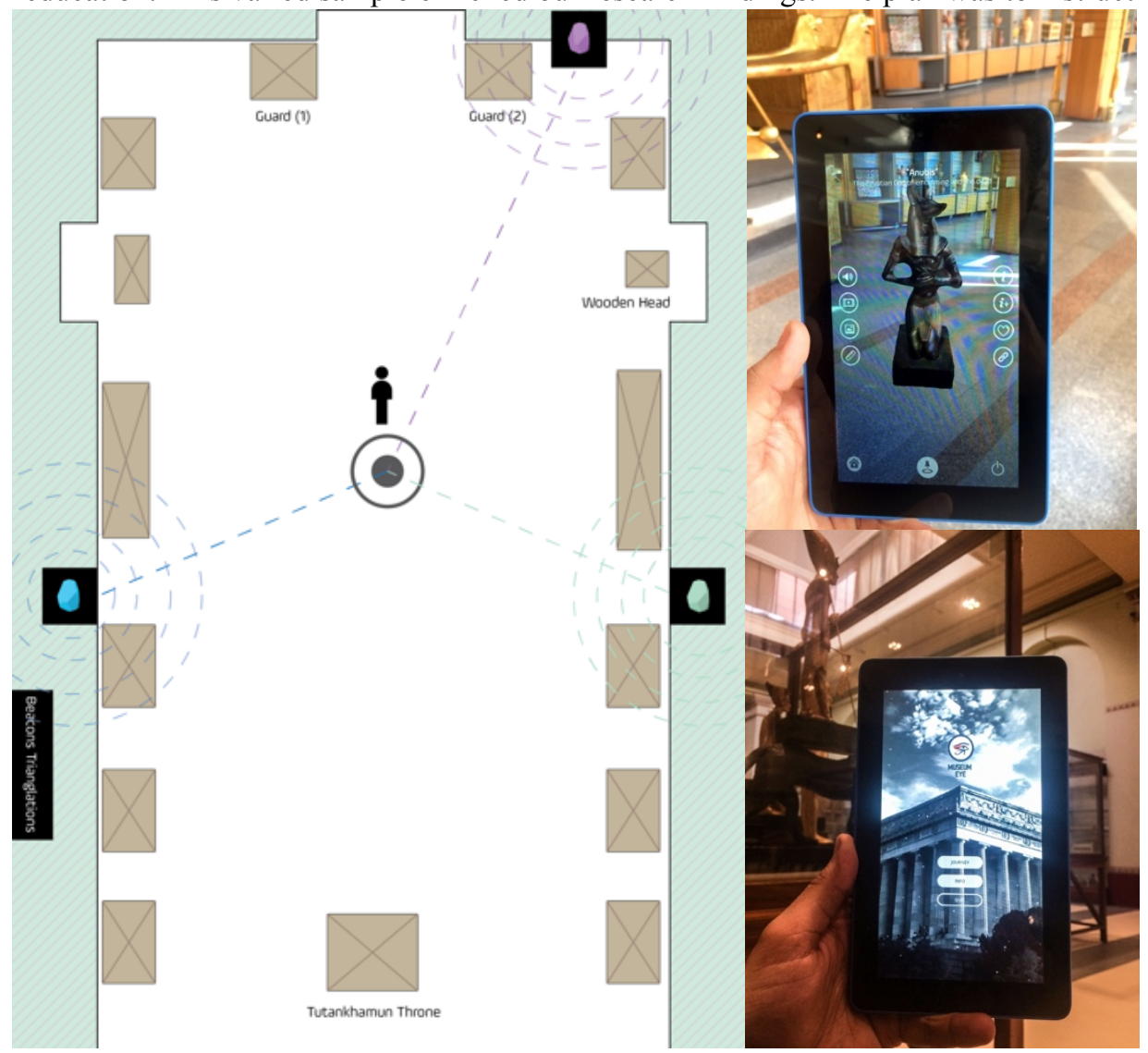

Figure 7 (left) Placing IBeacons in the Egyptian museum's room.

Figure 8: (Right) Testing MuseumEye at the Egyptian Museum in Cairo. 
the chosen participants to use the tablet containing the AR prototype during their regular visit. We briefly demonstrated the system to them individually, then they were asked to start their short tour taking the route described in the previous section. After finishing the planned tour, participants participated in a semi-structured questionnaire.

At the Egyptian museum in Cairo the system was setup in the eastern room in the application (product), centring around sense perceptions. While exploring the system in the targeted room, we captured viewers' emotions recognized through comments and facial expressions, and all of these observations were noted and analysed. These observations formed the basis of further questions to the users conducted verbally through interviews, in addition to their nonverbal questions in the questionnaires as depicted in fig.9.

Analysis of this user data led to substantial modifications. 'Visual Design': some alterations were accomplished in the interface design such as the button shapes, appearance and fonts. 'Interactivity aspect': the alterations included the navigation gestures and the flow of movement of the 3D model in the space to be more intuitive and familiar to potential users. The application was applied one more time in the Egyptian museum in Cairo, Egypt. At 'Stage 2', the researchers let the application be explored again by the Egyptian museum visitors. Through all of these phases, the application was enhanced to help it to improve on all of the UX aspects that were manifested before.

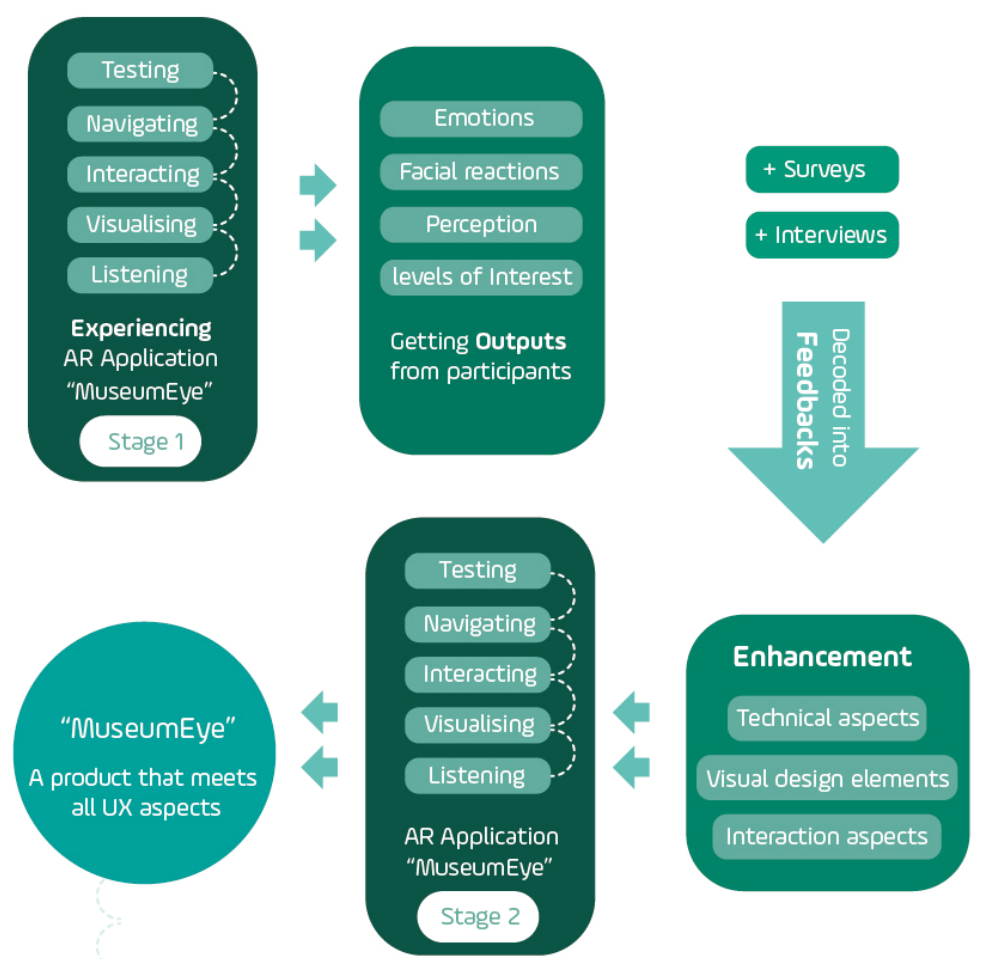

Figure 9 The pathway followed to build 'MuseumEye' 
Analysis of this user data led to substantial modifications. 'Visual Design': some alterations were accomplished in the interface design such as the button shapes, appearance and fonts. 'Interactivity aspect': the alterations included the navigation gestures and the flow of movement of the 3D model in the space to be more intuitive and familiar to potential users. The application was applied one more time in the Egyptian museum in Cairo, Egypt. At 'Stage 2', the researchers let the application be explored again by the Egyptian museum visitors. Through all of these phases, the application was enhanced to help it to improve on all of the UX aspects that were manifested before.

\section{$5 \quad$ Results}

After testing the application with 26 participants in Leeds museum and another 20 participants in the Egyptian museum in Cairo, the following results were collated, which present findings from the corresponding questionnaires. The majority of the responses were positive in both museums. In Leeds City Museum, the responses regarding the measured aspects are detailed below in the following table:

\begin{tabular}{|c|c|c|c|c|c|c|}
\hline Aspects & Strongly Agree & Agree & Neutral & Disagree & $\begin{array}{c}\text { Strongly } \\
\text { Disagree }\end{array}$ & $\begin{array}{c}\text { Average } \\
\text { (max =5) }\end{array}$ \\
\hline $\begin{array}{c}\text { Immersion } \\
\text { aspect }\end{array}$ & -- & $77 \%$ & $23 \%$ & -- & -- & 3.76 \\
\hline $\begin{array}{c}\text { Useful in } \\
\text { Guidance }\end{array}$ & $15 \%$ & $81 \%$ & $4 \%$ & -- & -- & 4.11 \\
\hline $\begin{array}{c}\text { System easy to } \\
\text { use }\end{array}$ & $19 \%$ & $69 \%$ & $8 \%$ & $4 \%$ & -- & 3.92 \\
\hline $\begin{array}{c}\text { Interesting in } \\
\text { museums }\end{array}$ & $19 \%$ & $77 \%$ & $4 \%$ & -- & -- & 4.15 \\
\hline Intuitive & $15 \%$ & $77 \%$ & $8 \%$ & -- & -- & 4.07 \\
\hline $\begin{array}{c}\text { Visuals served } \\
\text { the purpose }\end{array}$ & $8 \%$ & $92 \%$ & -- & -- & -- & 4.07 \\
\hline
\end{tabular}

Table 1: Shows the responses of Leeds museum visitors on 'MuseumEye' system'.

Overall, feedback from the testing phase suggested positive responses to the application as a means of providing guidance and information in the museum. Of the respondents of the Leeds Museum survey, $81 \%$ agreed that the application was useful in providing guidance to the museum's exhibits, with $77 \%$ agreeing that they felt immersed in the experience of using the application; that it was an interesting way of accessing information in the museum; and that it was intuitive to use.

$4 \%$ of respondents did not agree that the application was easy to use, and there were a number of low-scoring 'neither agree nor disagree' responses across all questions. 
These values have been taken into account to improve the functioning of the application for the next phase of evaluation conducted at the Egyptian Museum in Cairo. In the Egyptian Museum, the responses regarding the measured aspects are detailed below in the following table:

\begin{tabular}{|c|c|c|c|c|c|c|}
\hline Aspects & $\begin{array}{c}\text { Strongly } \\
\text { Agree }\end{array}$ & Agree & Neutral & Disagree & $\begin{array}{c}\text { Strongly } \\
\text { Disagree }\end{array}$ & $\begin{array}{c}\text { Average } \\
\text { (max =5) }\end{array}$ \\
\hline $\begin{array}{c}\text { Immersion } \\
\text { aspect }\end{array}$ & $55 \%$ & $45 \%$ & -- & -- & -- & 4.45 \\
\hline $\begin{array}{c}\text { Useful in } \\
\text { Guidance }\end{array}$ & $70 \%$ & $30 \%$ & -- & -- & -- & 4.7 \\
\hline $\begin{array}{c}\text { System easy to } \\
\text { use }\end{array}$ & $45 \%$ & $45 \%$ & $10 \%$ & -- & -- & 4.35 \\
\hline $\begin{array}{c}\text { Interesting in } \\
\text { museums }\end{array}$ & $19 \%$ & $77 \%$ & $4 \%$ & -- & -- & 4.45 \\
\hline Intuitive & $35 \%$ & $65 \%$ & -- & -- & -- & 4.35 \\
\hline $\begin{array}{c}\text { Visuals served } \\
\text { the purpose }\end{array}$ & $55 \%$ & $35 \%$ & $10 \%$ & -- & -- & 4.45 \\
\hline
\end{tabular}

Table 2: Shows the responses of the Egyptian museum visitors on 'MuseumEye' system.

After collating this information it became clear that the most significant different in responses was in relation to the question about the extent to which participants' felt that they were immersed in the experience of using the application. The scores increased in this area from an average of 3.76 out of 5 to an average of 4.45 out of 5 . This change may have resulted from the enhancements that were applied to the application after the initial testing phase at Leeds Museum, including amendments to the user interface and some functions relating to user interaction such as exploring the artifact in the $3 \mathrm{D}$ view.

It was also notable that the ease of use statement increased in this second testing phase from an average score of 3.92 to 4.35 out of 5, again as a result of enhancements in response to the initial testing phase.... The first version includes some gestures that users did not prefer such as navigation in the images gallery by clicking instead of swiping. Moreover, the level of 3D navigation freedom was very exaggerated. It was cumbersome to navigate the $3 \mathrm{D}$ object in 3 coordinates at the same time so we enhance it by freezing axis $\mathrm{Y}$ in order to navigate the $3 \mathrm{D}$ object around $\mathrm{X}$ and $\mathrm{Z}$. Other statements such as the usefulness and intuitiveness of the application also demonstrated an increased score from respondents. It appears from these responses that the enhancements made to the application for the second phase of testing had a beneficial effect on the overall functioning and usability of the application in its museum context.

These results helped to approve the validity of the system after its various stages of enhancements and, in turn, to support the validity of the UX framework that was designed specifically for this system.

The next two graphs show the potential of using Markerless Augmented Reality applications that use SLAM technology to guide visitors in museums, in comparison 
with the other alternative guides such as human guide, books and audio guides. Participants from Leeds museum chose the preferred guide as the AR guide with $42 \%$ against all other guidance methods. However, the analysis showed that the Egyptian museum's visitors chose the AR guide above all guides, with 50\% selecting AR as their preferred guide. These results were surprising to us as researchers, and contradicted our preliminary expectations. Conclusions can be drawn here about the likelihood of visitors to the Egyptian Museum potentially embracing MAR technology and using mobile devices in their tours (Figure 10).

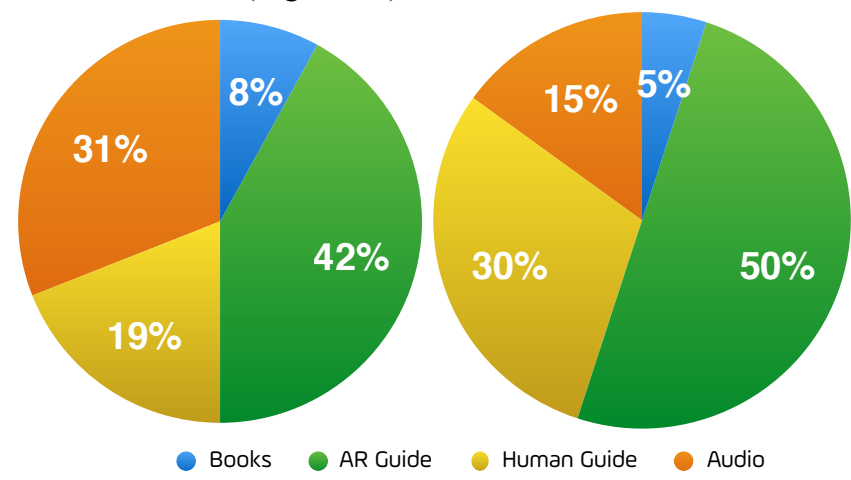

Figure 10: Guide methods chosen by Leeds Museum Visitors (Left) the Egyptian Museum Visitors (Right)

The Research conducted at Leeds City Museum and the Egyptian Museum, inCairo, indicates $77 \%$ of participants to have felt that using AR technology made the museumvisiting experience a more interesting one. It is interesting to consider the extent to which AR technology might enhance the visitor experience in terms of enjoyment by increasing interest and excitement. It is also important to consider the extent to which visitors' engagement with the process of using the technology might surpass and overshadow their engagement with - and understanding of/ learning taken from - the exhibits themselves. It seems fair and logical to suggest that excitement will be generated in the visitor by an interpretation device which is capable of 'bringing to life' an exhibit in such a way that the static object is shown to be kinetic and functioning in a new, dynamic context. The way in which it achieves this so effectively - and perhaps one of the most admirable functions of AR - is through its ability to blur the boundaries between reality and artifice. Perhaps the way AR appears to seamlessly meld the real and the illusory is so effective because of the fact that it is, in some respects, delivering reality: It delivers real, lived experience of one kind, in order to enhance real, lived experience of another kind. In this sense, the way it might potentially 'bring to life' an object is truly ground-breaking. The novelty and innovation of this technology's capabilities for animating exhibits in such a way is, arguably, likely to spark visitors' interest and excitement by providing a new type of encounter with the object or artefact, as well as, potentially, by enhancing their understanding and learning.

In light of the question of whether AR technology might limit human-to-human interaction and engagement in the museum, research conducted at both Leeds Museum 
and The Egyptian Museum, Cairo, asked visitors trialling AR technology to - the extent to which they considered it to actively enhance interaction between themselves and other visitors. Over $70 \%$ of participants at both museums agreed that the technology actually encouraged them to interact with others (see Figure 11). One reason for this might be the fact that, in being a novel form of interpretation, visitors were keen to discuss their experience of using the technology with others. The extent to which their interaction was to discuss their learning experience, or even the exhibits themselves, is unclear at this stage. It would be interesting to learn whether, if AR was to become an established form of interpretation in museums, this interaction would continue at such a level, or gradually subside.

In summary, AR presents exciting possibilities for museum interpretation. Its communicative capacity combines with its ability to potentially enhance what is seen and heard without having to substantially shift the viewer's field of vision away from an exhibit. This enables the viewer to retain an optimal vantage point for simultaneously viewing the exhibit and learning more about it. It also, potentially, enhances the viewer's understanding of the object's wider context by engaging them in a process of detailed interpretation - the extent to which it might affect visitors' knowledge and understanding of artefacts would be an interesting direction for further research into UX in AR in museums for MuseumEye.
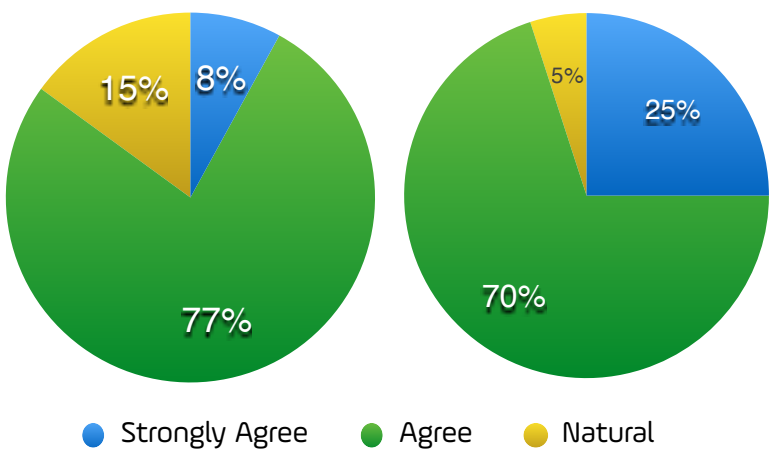

Figure 11: Responds on 'AR system encourages the human interaction' (Leeds museum on Left) (Egyptian museum on right)

It is also, however, important to remember that for some, new technology can be daunting and even alienating. For others, a more traditional museum-visiting experience might simply be preferred. Perhaps the most successful use of AR in museums would be to implement it as an option. By providing an element of choice, visitors would be able to access the technology or to opt for more familiar and established means of engaging with exhibits, depending upon their personal preferences. As a solution to museum interpretation, then, AR might be used in a way that reiterate's Prior's assertion that, 'Perhaps the most innovative and clear-sited 
museum directors are those who have recognised and exploited the plasticity of the museum idea in order to overlay various levels of aesthetic experience [33].

\section{Contribution}

This paper contributed theoretically by creating the characteristics of the user experience model for augmented reality applications in museums. The UX model process facilitates the procedures needed to identify the visitor's requirement in order to design and develop the system. Also, the UX system contributed to enhance the usability through two field studies and resulted an obvious enhancement on the museum experience. Additionally, the paper contributed practically through building a SLAM markerless AR mobile system 'MuseumEye' that integrated with iBeacons for the indoor spatial allocation purposes. The system surveyed in two museums and resulted positive responses on the usability aspects and for the overall museum experience during the usage of the system. The system passed through several enhancement during the evaluation process by the public contribution to assure the ultimate level of usability.

\section{Conclusion}

This paper has aimed to demonstrate a clear framework of the UX of markerless augmented reality application by manifesting the pathway that was approached for our system in both museums in Leeds, UK and Cairo, Egypt. It emphasized the user experience and usability aspects that should be considered when building a guided system for museums based around mobile devices. The framework has been applied in our system and assessed in two different museums in two different countries. Results show the potential of using AR guides in museums and demonstrate more positive results at the second museum after a series of developments had been put into place after recommended from the first museum visitors: The system was successfully improved based on these public contributions and the valuable feedback received.

Finally, this paper provides insight into the functionality and aesthetic aspects of the user experience of MAR applications which have an impact on the user's enjoyment, motivation, satisfaction and productivity while using the application. It also addresses the optimum levels of user interaction required to best experience the application, and, ultimately, to understand the relationship between AR systems and effective audience engagement in museums.

\section{Acknowledgments}

The authors would like to thank Leeds museum city staff and the Egyptian museum in Cairo for facilitating our activities. In addition, authors would also like to thank Helwan university and British Council in Egypt and the Egyptian Culture Bureau as the main funding body for our research and all who supported us and gave us the valuable advises to design and develop our system. 


\section{References}

[1] Azuma, R.T., A survey of augmented reality. Presence, 1997. 6(4): p. 355-385.

[2] Azuma, R., et al., Recent advances in augmented reality. IEEE computer graphics and applications, 2001.21(6): p. 34-47.

[3] Höllerer, T. and S. Feiner, Mobile augmented reality. 2004.

[4] Damala, A., I. Marchal, and P. Houlier. Merging augmented reality based features in mobile multimedia museum guides. in Anticipating the Future of the Cultural Past, CIPA Conference 2007, 1-6 October 2007. 2007.

[5] Wojciechowski, R., et al., Building Virtual and Augmented Reality museum exhibitions, in Proceedings of the ninth international conference on 3D Web technology. 2004, ACM: Monterey, California. p. 135-144.

[6] Tscheu, F. and D. Buhalis, Augmented Reality at Cultural Heritage sites, in Information and Communication Technologies in Tourism 2016. 2016, Springer. p. 607-619.

[7] Teichrieb, V., et al., A survey of online monocular markerless augmented reality. International Journal of Modeling and Simulation for the Petroleum Industry, 2007. 1(1).

[8] Zhou, F., H.B.-L. Duh, and M. Billinghurst. Trends in augmented reality tracking, interaction and display: A review of ten years of ISMAR. in Proceedings of the 7th IEEE/ACM International Symposium on Mixed and Augmented Reality. 2008. IEEE Computer Society.

[9] Molton, N., A.J. Davison, and I. Reid. Locally Planar Patch Features for Real-Time Structure from Motion. in BMVC BRITISH MACHINE VISION CONFERENCE. 2004.

[10] Davison, A.J. Real-time simultaneous localisation and mapping with a single camera. in Computer Vision, 2003. Proceedings. Ninth IEEE International Conference on. 2003. IEEE.

[11] Eade, E. and T. Drummond. Edge landmarks in monocular SLAM. in In Proc. British Machine Vision Conf. 2006. Citeseer.

[12] Hammady, R., M. Ma, and N. Temple. Augmented Reality and Gamification in Heritage Museums. in Joint International Conference on Serious Games. 2016. Springer.

[13] Chekhlov, D., et al. Ninja on a plane: Automatic discovery of physical planes for augmented reality using visual slam. in Proceedings of the 2007 6th IEEE and ACM International Symposium on Mixed and Augmented Reality. 2007. IEEE Computer Society.

[14] Neubert, J., J. Pretlove, and T. Drummond. Semi-autonomous generation of appearancebased edge models from image sequences. in Proceedings of the 2007 6th IEEE and ACM International Symposium on Mixed and Augmented Reality. 2007. IEEE Computer Society.

[15] Comport, A.I., et al., Real-time markerless tracking for augmented reality: the virtual visual servoing framework. IEEE Transactions on visualization and computer graphics, 2006. 12(4): p. 615-628.

[16] Proctor, N., Off base or on target? Pros and cons of wireless and location-aware applications in the museum. ICHIM, Paris, France, 2005.

[17] Azuma, R.T., A survey of augmented reality. Presence: Teleoperators and virtual environments, 1997. 6(4): p. 355-385.

[18] Barandiaran, I., C. Paloc, and M. Graña, Real-time optical markerless tracking for augmented reality applications. Journal of Real-Time Image Processing, 2010. 5(2): p. $129-138$.

[19] Newcombe, R.A., et al. KinectFusion: Real-time dense surface mapping and tracking. in Mixed and augmented reality (ISMAR), 2011 10th IEEE international symposium on. 2011. IEEE.

[20] Morrison, A., et al. Like bees around the hive: a comparative study of a mobile augmented reality map. in Proceedings of the SIGCHI Conference on Human Factors in Computing Systems. 2009. ACM. 
[21] Tillon, A.B., et al. A day at the museum: An augmented fine-art exhibit. in 2010 IEEE International Symposium on Mixed and Augmented Reality-Arts, Media, and Humanities. 2010. IEEE.

[22] Damala, A. and N. Stojanovic. Tailoring the Adaptive Augmented Reality (A 2 R) museum visit: Identifying Cultural Heritage professionals' motivations and needs. in 2012 IEEE International Symposium on Mixed and Augmented Reality-Arts, Media, and Humanities (ISMAR-AMH). 2012. IEEE.

[23] Krzych, J. Estimote Beacons. 2015 [cited 2016; Available from: http://estimote.com/.

[24] Law, E.L.-C., et al. Understanding, scoping and defining user experience: a survey approach. in Proceedings of the SIGCHI conference on human factors in computing systems. 2009. ACM.

[25] ISO. Ergonomics of human-system interaction - Part 210: Human-centred design for interactive systems. 2010 [cited Feb 2017; Available from: https://www.iso.org/obp/ui/ iso:std:iso:9241:-210:ed-1:v1:en.

[26] Ellis, J.C.K.Z.S.N.M., UX Design 2015 \& 2016: successful Trends for digital Products. 2015: UXPin inc.

[27] Unger, R. and C. Chandler, A Project Guide to UX Design: For user experience designers in the field or in the making. Second Edition ed. 2012: Pearson Education.

[28] Hassenzahl, M. and N. Tractinsky, User experience-a research agenda. Behaviour \& information technology, 2006. 25(2): p. 91-97.

[29] Forlizzi, J. and K. Battarbee. Understanding experience in interactive systems. in Proceedings of the 5th conference on Designing interactive systems: processes, practices, methods, and techniques. 2004. ACM.

[30] Würstl, D. User Experience consists of Usability, Look and Feel. 2009; Available from: http://www.katzenbergdesign.net/.

[31] Dam, R.F. and T.Y. Siang. Learn How to Use the Best Ideation Methods: SCAMPER 2017 [cited 2017; Available from: https://www.interaction-design.org/literature/article/learnhow-to-use-the-best-ideation-methods-scamper.

[32] Dam, R.F. and T.Y. Siang. Introduction to the Essential Ideation Techniques which are the Heart of Design Thinking. 2017 [cited 2017; Available from: https://www.interactiondesign.org/literature/article/introduction-to-the-essential-ideation-techniques-which-arethe-heart-of-design-thinking.

[33] McClellan, A., Art and Its Publics: Museum Studies at the Millennium. 2008: Wiley. 